\title{
Urban Public Safety Risk Assessment of 35 Cities in China Based on Context-Dependent DEA Approach And DEASort
}

\author{
Ying-ying Zeng \\ Wuhan University of Technology, Wuhan, China
}

\begin{abstract}
Urban public safety is the foundation of the urban development. Correct and reasonable assessment of urban public safety plays a vital role in the healthy development of cities. This paper establishes a context-dependent DEA model to divide 35 cities of China into three levels, and considers improved value of the low-level when different contexts are used as reference backgrounds. Then use the idea of DEASort proposed by Alessio Ishizaka to prioritize the high level in combination with the index weights in the Public Safety Blue Book: China Urban Public Safety Development Report (2016-2017). The research shows that there is a significant stepwise difference in urban public safety of China: the public security situation in the eastern cities is relatively optimistic; the levels in the northeastern cities are basically the same, and there is still room for improvement; the central cities are stratified, some cities are relatively good, and some cities have larger improve space; there are major hidden dangers in the public security problems in western cities.
\end{abstract}

\section{Introduction}

With the increasing density and speed of urban development, urban public safety is becoming more and more important as the cornerstone of urban management. Some countries began to take emergency precautions against urban public safety at the end of the 18th century. In 1979, the United States established the Federal Emergency Management Agency; In 1995, Japan established an early evaluation system for earthquake disaster prevention and emergency response support system; In 1992, the United Kingdom established an emergency command system and set up an emergency platform in the local government. However, the development of urban public security construction in China is relatively late. Since 2003, as an important aspect of national planning, public security construction has been supported in many aspects and made great progress on the premise of null foundation. However, our country concentrates mostly on the level of general disaster prevention and mitigation and emergency management, and there is still much room for theoretical system.

At present, in the study of urban public safety risk assessment, Liu Chengshui et al. [1] used factor analysis and fuzzy neural network to evaluate the level of urban public safety, which provided a scientific supplement to traditional methods by emphasizing energy saving at the level of fuzzy rules; Sun Huali et al. [2] based on fishbone diagram analysis, established an index system, and used principal component analysis to measure public safety risk. Zhang Jiaming et al. [3] constructed and studied the evaluation system tentatively by combining qualitative and quantitative methods, and selected several factors to get the final evaluation results by analytic hierarchy process. Yang Ruihan et al. [4] introduced group decision into FHAP, and used the combination of group decision and FAHP to construct the multi-level extension evaluation model of urban public safety. Wang Yibao et al. [5] used analytic hierarchy process, entropy weight method and coefficient of variation method to determine subjective and objective weights, applied relative entropy principle to combine weights, and used grey correlation analysis to calculate the correlation degree. Finally, based on the evaluation mathematical model of MATLAB, evaluated and analyzed the situation of urban public safety in China.

Through the above research, we can see that most scholars pay attention to the scientific construction of system indicators, lacking of attention to the expansion and innovation of theoretical methods in empirical evaluation model. And the results are mostly based on the absolute evaluation value of theory, short of comparison of relativity between cities. Based on these issues, this paper takes the data of 35 cities of China in 2017 as an example, constructs the index system in the Blue Book of Public Safety: Report on the Development of Urban Public Safety in China (2016-2017)[6] as a reference, and then establishes a reasonable index system according to the actual situation. Finally, the auther introduces the situational dependence model to divide 35 cities into three best practice frontiers as evaluation scenarios, and raises the low-level cities base on the improved value. For different scenarios, we can use DEASort methodology for reference, and refer to the index weight in the Blue Book of Public Safety: China Urban Public Safety Development Report (2016-2017). We can distribute the weight of the index system in this paper, and get the results of high-level 
internal classification, which can provide reference for the development of urban public safety.

\section{Contex-dependent DEA modle and Fixed-weight DEASort Methodology}

\subsection{Context-Dependent DEA Model}

In the original DEA method, the efficiency of each decision-making unit is evaluated by comparing with the decision-making unit on the frontier of best practice. However, this traditional method has some limitations in some respects: (1) For the decision-making unit on the frontier of best practice, it is impossible to decide the proficiency. Even if the super-efficiency model can not get it due to the difference of reference sets leads to evaluation errors. (2) The change of some ineffective decision making units will not affect other ineffective decision making units. That is, the change of environment (except the best frontier) will not affect the decision making units, which is obviously inconsistent with the actual cognition. Therefore, Seiford and Zhu expand the context-dependent DEA model [7] on the basis of the original DEA, dividing the frontier of best practice into several levels, and get the improved value of different decision-making units under different situations.

Define $J^{l}=\left\{D M U_{j}, j=1, \ldots, n\right\}$ (including a set of $\mathrm{N}$ decision units) and iterative definition $J^{l+1}=J^{l}-E^{l}$, where $E^{l}=\left\{D M U_{k} \in J^{l} \mid \theta^{*}(l, k)=1\right\}$ is the best practice frontier of level 1 and $\theta^{*}(l, k)$ is the optimal value of $D M U_{k}$ based on input-oriented CRS model, according to which the rules are as follows:

$$
\theta^{*}(\mathrm{l}+\mathrm{k})=\min _{\lambda_{j, \theta(l, k)}} \theta(l, k)
$$

s.t.

$$
\begin{gathered}
\sum_{j \in F\left(J^{l}\right)} \lambda_{j} x_{i j} \leq \theta(l, k) x_{i k} \\
\sum_{j \in F\left(J^{l}\right)} \lambda_{j} y_{r j} \geq y_{r k} \\
\lambda_{j} \geq 0, j \in F\left(J^{l}\right)
\end{gathered}
$$

$E^{l}$ is the best practice frontier of level 1, and $\theta^{*}(\mathrm{l}+\mathrm{k})$ is the optimal value of $D M U_{k}$ based on inputoriented CRS model.

Consider the following measures: $D M U_{q} \in E^{l_{0}}, l_{0} \in$ $\{2, \ldots, L\}$, the linear programming of the improved value is as follows:

$$
G_{q}^{*}(g)=\min _{\lambda_{j}, G_{q}(g)} G_{q}(g) \quad g=1, \ldots, l_{0}-1
$$

s.t.

$$
\begin{gathered}
\sum_{j \in F\left(E^{\left.l_{0}-g\right)}\right.} \lambda_{j} x_{j} \leq G_{q}(\beta) x_{q} \\
\sum_{j \in F\left(E^{l_{0}-g}\right)} \lambda_{j} y_{j} \geq y_{q} \\
\lambda_{j} \geq 0 \quad j \in F\left(E^{l_{0}-g}\right)
\end{gathered}
$$

Here is $\mathrm{G}_{\mathrm{q}}^{*}(g+1)<G_{q}^{*}(g)$, which is called $M_{q}^{*}=$ $1 / G_{q}^{*}$ as the g-level improvement of $D M U_{q}$ in the hierarchical $E^{l_{0}}$.

\subsection{Fixed-Weight DEASort Methodology}

In the context-dependent DEA model, the attractiveness measure is proposed to distinguish the decision-making units with the same performance. The author thinks that the hierarchical decision-making units can be fully combined with the hierarchical and intra-level differences. When evaluating the low-level units, the high-level units can be used as reference sets. When evaluating the highlevel units, the classification method of DEASort (Alessio Ishizaka) [8] can be used as reference sets, and other decision-making units at this level can be used as reference sets. According to the existing weights, the decision-making units at this level can be imported internally. Row classification can be roughly divided into the following steps:

Step 1 Normalisation of item scores

The measured score $v_{i j}$ of each item for each criterion $\mathrm{j}$ is normalized on a $0-1$ scale to make them comparable via the following expression:

$$
v_{i, j}^{*}=\frac{v_{i, j}-\min _{i=1, \ldots, I} v_{i, j}}{\max _{i=1, \ldots, I} v_{i, j}-\min _{i=1, \ldots, I} v_{i, j}} \quad \forall i=1, \ldots, I
$$

Step2 Criteria weight evaluation

According to the established index system and the weight of objective index system of public safety in the Blue Book of Public Safety: China Urban Public Safety Development Report (2016-2017), the weight of selected index is redefined. According to the weight, the index importance matrix is obtained. 1 indicates equal importance and 9 indicates extrem importance. Vector of weights are founded by calculating the eigenvector(Satty,1980).

$$
A_{k} w_{k}=\lambda_{\operatorname{maxk}} w_{k}
$$

$A_{k}$ is the important degree matrix and $w_{k}$ is the newly allocated weight vector.

Step 3 Calculation of the item priority

For each specific item o under evaluation,its priority can be calculated:

$$
P_{0}=\sum_{j=1}^{J} w_{j} v_{o j}
$$

s.t.

$$
\begin{gathered}
\sum_{j=1}^{J} w_{j} v_{i j} \leq 1 \quad i=1, \ldots, I \\
w_{j} \geq 0 \quad i=1, \ldots, I
\end{gathered}
$$

Step 4 Sorting into classes

Set threshold according to the overall numerical distribution of priority. The rules are as follows:

$$
\begin{gathered}
P_{z} \geq t h_{1} \Rightarrow k \in C_{1} \\
t h_{2} \leq P_{z} \leq t h_{1} \quad \Rightarrow k \in C_{2} \\
\cdots \\
P_{z}<t h_{n-1} \Rightarrow k \in C_{n}
\end{gathered}
$$

\section{Empirical Study on Public Safety Risk Analysis of 35 Cities in China}

Thirty-five cities in China are selected as evaluation objects. Firstly, the contaxt-dependent method is used to stratify them, and then the improved values of low-level decision-making units in different situations are obtained. 
Finally, DEASort method is used to classify high-level units.

\subsection{Rating Indicators and Data Sources}

Referring to the objective index system of public safety in the Blue Book of Public Safety: Report on the Development of Urban Public Safety in China (20162017), this paper obtains the corresponding input and output indicators, as shown in Table 2. The input index of urban public security risk assessment system is constructed from two aspects of social security and public health, and the output index is constructed from three aspects of social security, public health and accident disaster. The data in this paper are from EPS database [9], China Urban Statistics Yearbook 2018[10], China Insurance Yearbook 2016[11] and China Health Statistics Yearbook 2018[12].

\subsection{Hierarchical DEA Hierarchical Results and Improvement Measure}

Through the situation dependent DEA model, 35 cities are stratified, and the following hierarchical results are obtained by using Excel solver.

$E^{1}=\left\{\mathrm{DMU}_{\mathrm{q}} \mid \mathrm{q}=\right.$ Beijing, Tianjin, Shi jiazhuang,

Huhhot, Shenyang, Dalian, Changchun, Harbin,

Shanghai, Nanjing, Hefei, Qingdao, Jinan, Zhengzhou,

Changsha, Shenzhen, Nanning, Haikou, Yinchuan\}; $E^{2}=\left\{\mathrm{DMU}_{\mathrm{q}} \mid \mathrm{q}=\right.$ Taiyuan, Hangzhou, Ningbo,

Fuzhou, Xiamen, Nanchang, Wuhan, Guangzhou, Chongqing, Chengdu, Guiyang, Kunming, Xi'an, Lanzhou, Xining\};

$$
E^{3}=\left\{\mathrm{DMU}_{\mathrm{q}} \mid \mathrm{q}=\text { Urumqi }\right\}
$$

Regarding the decision-making units at $E^{2}$ and $E^{3}$ levels as the low level, and the decision-making units at $E^{1}$ level as the high level, we can get the measure of the improvement of 16 low-level objects in the situation of $E^{1}$ in table 1,the ranking is as follows: Xiamen, Wuhan, Urumqi, Lanzhou, Taiyuan, Guangzhou, Chongqing, Kunming, Guiyang, Xi'an, degree, Nanchang, Ningbo, Xining, Fuzhou. Hangzhou.

Table 1. Improvement value on Level 1

\begin{tabular}{cccr}
\hline \multirow{2}{*}{ City } & \multicolumn{3}{c}{ Level1 as the context } \\
\cline { 2 - 4 } & $\begin{array}{c}\text { Related } \\
\text { Efficiency }\end{array}$ & $\begin{array}{c}\text { Improved } \\
\text { value }\end{array}$ & Ranking \\
\hline Xiamen & 0.5573 & 1.7944 & 1 \\
Wuhan & 0.6201 & 1.6126 & 2 \\
Urumqi & 0.6266 & 1.5959 & 3 \\
Lanzhou & 0.7305 & 1.3689 & 4 \\
Taiyuan & 0.7428 & 1.3463 & 5 \\
Guangzhou & 0.7843 & 1.275 & 6 \\
Chongqing & 0.7957 & 1.2568 & 7 \\
Kunming & 0.7966 & 1.2553 & 8 \\
Guiyang & 0.8598 & 1.1631 & 9 \\
Xi'an & 0.8619 & 1.1602 & 10 \\
Chengdu & 0.8633 & 1.1583 & 11 \\
Nanchang & 0.9121 & 1.0964 & 12
\end{tabular}

\begin{tabular}{crrr} 
Ningbo & 0.9174 & 1.09 & 13 \\
Xining & 0.9246 & 1.0815 & 14 \\
Fuzhou & 0.9352 & 1.0693 & 15 \\
Hangzhou & 0.9498 & 1.0529 & 16 \\
\hline
\end{tabular}

\subsection{Classification with Fixed-Weight DEASort}

\subsubsection{Normalization}

The indexes of 35 cities were normalized one by one, and the standard values of all data were obtained.

\subsubsection{Matrix of Importance}

According to the selected index and referring to the weight of objective index system of public safety in Blue Book of Public Safety: Report on the Development of Urban Public Safety in China (2016-2017), the weight number of the index in this paper is obtained, and the importance matrix of the output index is compared according to the weight.

Table 2. Urban Public Safety Index System

\begin{tabular}{|c|c|}
\hline & Indicators \\
\hline Input & $\begin{array}{l}\text { Population Density }\left(\text { Person/Km}{ }^{2}\right) \\
\text { Unemployment rate }(\%) \\
\text { Number of Institutions of Higher Education per } \\
10000 \text { People } \\
\text { Number of Hospitals (Institutions) per } 10,000 \\
\text { People } \\
\text { Insurance Density (RMB 10,000) } \\
\text { Environment Pollution Control Investment } \\
\text { Proportion of GDP } \\
\text { Per Capita Public Security Financial Expenditure } \\
\text { (RMB 10,000) } \\
\text { Unemployment Insurance Participation Rate (\%) } \\
\text { Class A and B Infectious Disease Incidence Rate } \\
(1 / \%)\end{array}$ \\
\hline Output & $\begin{array}{l}\text { Per Capita Gross Domestic Product (RMB) } \\
\text { Personal Insurance Expenditure Compensation } \\
(1 / 100 \text { yuan) } \\
\text { Number of Traffic Casualties per } 10,000 \text { People }\end{array}$ \\
\hline
\end{tabular}

Table 3. One Classification Result

\begin{tabular}{lrrll}
\hline A & & & B & \\
\cline { 1 - 2 } \cline { 5 - 5 } Item & score & & Item & score \\
\hline Beijing & 0.984 & & Shenyang & 0.476531 \\
Yinchuan & 0.9676 & & Dalian & 0.398218 \\
Haikou & 0.961759 & & Changsha & 0.379136 \\
Shanghai & 0.958185 & & Shijiazhuang & 0.365917 \\
Huhhot & 0.802856 & & Changchun & 0.353736 \\
Tianjin & 0.703022 & & Harbin & 0.318643 \\
Nanjing & 0.661496 & & Nanning & 0.23196 \\
Shenzhen & 0.610771 & & \\
Qingdao & 0.568614 & & & \\
Heifei & 0.556618 & & \\
Zhengzhou & 0.519523 & & \\
Jinan & 0.50025 & & \\
\hline
\end{tabular}




\subsubsection{Assign to Class}

According to the processing of MATLAB, the feature vectors which conform to the model constraints can be obtained, and the score can be obtained by processing. According to the actual requirements and the experience of decision makers, threshold can be set. If 0.5 is used as the only threshold, 19 cities in Level 1 can be divided into $\mathrm{A}$ and $\mathrm{B}$ categories, shown in Table 3.

\section{Analysis and conclusion of results}

Within the scope of the indicators, the following conclusions can be drawn through the context-dependent model and Fixed-Weight DEASort model: (1) The results of public safety risk assessment of the selected representative 35 cities show obvious geographical stratification, the eastern cities are relatively ideal; the northeastern cities are next; the central cities are divided separatly; and there are big hidden dangers in the the western cities .(2) The selected northeastern cities belong to the category B in high-level, which indicates that the northeastern cities are in the bottleneck period of building a safe city. (3) The overall risk coefficient of western cities is great, and the improvement space is larger than that of eastern and northeastern cities, but some cities perform better in individual indicators.

Based on the above analysis, cities in different regions should adopt different strategies to reduce the risk of urban public safety and maintain the healthy development of cities. Central cities can adopt the method of "one area saves many areas" to balance the problem of differentiation; northeastern cities should adopt new strategies to break through the bottleneck in maintaining urban public security; western cities should break through one by one and learn from each other.

\section{Reference}

1. Liu.Chengshui, JBCC, Study on urban public safety evaluation based on factor analysis and fuzzy neural network, 1,7(2010)

2. Sun.Huali, CSSJ, Risk Measurement and Evaluation of Public Safety Based on Fishbone Diagram,21,6(2011)

3. Zhang.Jiaming, HRE, Evaluation of urban community public safety based on AHP (2019)

4. Yang.Ruihan, JSST, Evaluation of urban public safety based on group decision - making and fuzzy analytic hierarchy process, 11,7(2015)

5. Wang.Yibao, SDM, Probabilistic Hesitant Fuzzy Multi-attribute Decision-making Method Based on Correlation Coefficient,18,6 (2019)

6. Huang. Yuhua, Yang. Wenming, Zhao. Jianhui, Blue Book of Public Safety: Report on the Development of Urban Public Safety in China (2016-2017) (2017)

7. Wade D.Cook, Zhu.Joe, Balanced Benchmarking Method for Data Envelopment Analysis(2017)

8. Alessio. Ishizaka, IJPE, DEASort: Assigning items with data envelopment analysis in ABC classes (2018)

9. EPS database. http: //olap.epsnet.com.cn/

10. Chen. Xiaolong, China City Statistical Yearbook (2018)

11. Zhou.Yanli, Yearbook Of China's Insurance (2016)

12. Zhang.Xuegao, China Health Statistics Yearbook (2018) 